\title{
Lymphocyte antigens targetable by monoclonal antibodies in non-systemic vasculitic neuropathy
}

\author{
Christian Schneider, ${ }^{1}$ Gilbert Wunderlich, ${ }^{1}$ Johannes Bleistein ${ }^{2}$ Gereon R Fink, ${ }^{1,3}$ \\ Martina Deckert, ${ }^{4}$ Anna Brunn, ${ }^{4}$ Helmar Christoph Lehmann ${ }^{1}$
}

${ }^{1}$ Department of Neurology, University of Cologne, Köln, Germany

${ }^{2}$ Department of Neurology, St. Katharinen Hospital, Frechen, Germany

${ }^{3}$ Institute of Neuroscience and Medicine (INM-3), Research Centre Juelich, Jülich, Germany ${ }^{4}$ Department of Neuropathology, University of Cologne, Köln, Germany

\section{Correspondence to}

Dr Helmar Christoph Lehmann Department of Neurology, University Hospital of Cologne, Kerpener Straße 62, Köln D-150937, Germany; helmar. lehmann@uk-koeln.de

$\mathrm{AB}$ and $\mathrm{HCL}$ contributed equally.

Received 17 February 2017 Revised 21 April 2017

Accepted 3 May 2017 Published Online First 27 May 2017

\section{CrossMark}

\section{To cite: Schneider $C$} Wunderlich G, Bleistein J, et al. J Neurol Neurosurg Psychiatry 2017:88:756-760.

\section{ABSTRACT}

Objective To identify the most relevant antigens for monoclonal antibodies in lymphocytic infiltrates in nonsystemic vasculitic neuropathy (NSVN).

Background Current immunosuppressive treatment for NSVN is insufficient. Monoclonal antibodies might be a treatment option, but the expression profile for targetable antigens on lymphocytic infiltrates in NSVN is unknown.

Methods Sural nerve biopsies from a cohort of patients with NSVN were immunohistochemically studied for the expression of potential candidate antigens in perivascular and intramural lymphocytic infiltrates and correlated with neurological and electrophysiological parameters. 20 patients with treatment naïve NSVN and 5 patients with idiopathic axonal neuropathy were included. Results The CD52, BAFF and CD49d antigens were expressed in epineurial, perivascular or intramural lymphocytes of all (20/20) patients. CD52 was most prominently expressed in $21.49 \%$ of all inflammatory infiltrates. BAFF and CD49d were detected in 11.25\% and $10.99 \%$ of these lymphocytes, respectively. The CD20, CD25 and CD126 antigens were found less frequently and at low levels only (CD20: 10/20 patients, $5.84 \%$ of lymphocytes; CD25: $17 / 20$ patients, $5.22 \%$ of lymphocytes; CD126: $3 / 20$ patients, $0.15 \%$ of lymphocytes).

Conclusion This is the first study in NSVN that identifies antigens expressed by pathogenic lymphocytes, which are potential targets for future monoclonal antibody treatment. Our data suggest that NSVN is amenable to monoclonal antibodies and, moreover, that targeting CD52 may be particularly promising. Our results strongly warrant future clinical trials in NSVN with monoclonal antibodies.

\section{INTRODUCTION}

Non-systemic vasculitic neuropathy (NSVN) is a frequent cause of chronic progressive axonal peripheral neuropathy. Diagnosis is established by nerve biopsy, which demonstrates axonal damage with asymmetric loss of nerve fibres. Perivascular inflammatory infiltrates invade blood vessel walls resulting in haemorrhage and necrosis, followed by vascular obliteration. ${ }^{1}$ Due to a lack of controlled trials in NSVN, current treatment recommendations are based on expert opinions or the extrapolation of results from treatment trials in systemic vasculitis. ${ }^{2}$ ${ }^{3}$ However, the concept that NSVN may constitute a separate disease entity challenges the validity of the latter approach. ${ }^{14}$ It is assumed that NSVN is amenable to immunosuppressive treatment. ${ }^{15}$ So far, treatment regimens include corticosteroids, usually in combination with cyclophosphamide in order to avoid the high relapse rates of corticoid monotherapy. Frequently, this combined immunosuppression needs to be discontinued due to severe side effects such as pneumonia, sepsis or leucopenia. $^{6}$

The identification of molecules that are pathogenetically relevant and that may be targeted specifically by recombinant monoclonal antibodies opens an exciting novel strategy in the therapy of autoimmune diseases. Such an approach may also yield novel therapeutic concepts for NSVN. So far, monoclonal antibodies directed against cytokines (B cell activating factor, BAFF), cytokine receptors, such as cluster of differentiation (CD) 25 and CD126 and other surface molecules(CD20, CD52) are currently tested or have already been approved for treating immune-mediated neurological diseases such as multiple sclerosis. ${ }^{7-11}$ In contrast, such a strategy has not yet been evaluated in NSVN.

To pave the way for studies with monoclonal antibodies in NSVN, we here characterised the phenotypic profile of inflammatory infiltrates in a series of 20 sural nerve specimens from patients with NSVN. We focused on immunologically relevant molecules for which Food and Drug Administration-approved monoclonal antibodies are available.

Our study identifies CD52 as the most frequently and prominently expressed antigen in NSVN. Thus, the use of antibodies, which target CD52, for the treatment of NSVN warrants further investigation.

\section{MATERIALS AND METHODS}

Clinical and electrophysiological assessment of patients

Clinical data and biopsy specimens from 20 untreated patients with NSVN were analysed retrospectively. All patients fulfilled the diagnostic criteria for pathologically definite NSVN according to the guidelines of the Peripheral Nerve Society. ${ }^{1}$ There was no evidence for systemic vasculitis, neither clinically nor in laboratory tests. For control, clinical data and biopsy specimens obtained from five patients with chronic idiopathic non-inflammatory axonal neuropathy were included. In addition, sural nerve biopsies of two patients each suffering from anti-neutrophil cytoplasm antibody-associated systemic vasculitis (AASV) and chronic inflammatory demyelinating polyneuropathy (CIDP), respectively, as well as temporal artery biopsies of two 
Table 1 Primary antibodies used for immunohistochemistry

\begin{tabular}{|c|c|c|c|c|c|}
\hline Antibody & Clone & Species & Dilution & Heat-induced epitope retrieval & Company \\
\hline LCA (CD45) & $\mathrm{X} 16 / 99$ & Mouse & 1:50 & Citrate buffer $\mathrm{pH} 6.0$ & Leica, Wetzlar, Germany \\
\hline CD3 & LN10 & Mouse & $1: 200$ & Target buffer pH 9.0 & Leica \\
\hline $\mathrm{CD} 4$ & $4 B 12$ & Mouse & $1: 40$ & Target buffer $\mathrm{pH} 9.0$ & Biogenex, DCS, Hamburg, Germany \\
\hline CD8 & $C 8 / 144 B$ & Mouse & $1: 200$ & Target buffer $\mathrm{pH} 9.0$ & Covance, München, Germany \\
\hline CD68 & $514 \mathrm{H} 12$ & Mouse & $1: 1000$ & Target buffer $\mathrm{pH} 9.0$ & Leica \\
\hline CD52 & YTH34.5 & Rat & 1:500 & Target buffer pH 9.0 & Serotec, Puchheim, Germany \\
\hline CD25 (IL-2R) & $4 \mathrm{C} 9$ & Mouse & $1: 25$ & Target buffer pH 9.0 & Leica \\
\hline BAFF & Buffy 2 & Rat & $1: 200$ & Target buffer $\mathrm{pH} 9.0$ & Enzo Life Sciences, Lörrach, Germany \\
\hline CD20 & L26 & Mouse & $1: 300$ & Citrate buffer $\mathrm{pH} 6.0$ & Leica \\
\hline CD49d (VLA-4) & Polyclonal & Rabbit & $1: 40$ & Target buffer pH 9.0 & Serotec \\
\hline CD126 (IL-6R) & B-R6 & Mouse & $1: 5$ & Target buffer $\mathrm{pH} 9.0$ & Serotec \\
\hline
\end{tabular}

LCA, leucocyte common antigen. VLA, very late antigen.

patients suffering from temporal arteritis have been included. All patients were examined neurologically and electrophysiologically including standard nerve conduction studies. ${ }^{12}$ The study protocol was approved by the institutional review board of the University Hospital Cologne, Cologne, Germany (protocol number 16-285).

\section{Nerve biopsy and immunohistochemical profiling}

All patients underwent sural nerve biopsy after obtaining written informed consent. Nerve specimens were subdivided into three parts: one fraction was snap frozen in isopentane (Fluka, Neu-Ulm, Germany), precooled by liquid $\mathrm{N}_{2}$ and stored at $-80^{\circ} \mathrm{C}$ until preparation of $9 \mu \mathrm{m}$ thick sections; the second fraction was formalin fixed and paraffin embedded to prepare $4 \mu \mathrm{m}$ thick sections; the third fraction was fixed in $3.9 \%$ phosphate buffered glutaraldehyde (Merck, Darmstadt, Germany) for 48 hours, osmicated and embedded in epoxy resin (Serva, Heidelberg, Germany) for preparation of $1 \mu \mathrm{m}$ semithin sections. Frozen sections were stained with H\&E; enzyme histochemistry was performed with Engel's modified trichrome staining. Semithin sections were stained with $0.1 \%$ toluidine blue $\mathrm{O}$ (Merck) in 2.5\% sodium carbonate (Merck). Formalin-fixed, paraffin-embedded nerve specimen sections were stained with Prussian blue and used for immunohistochemistry employing the avidin-biotin complex technique with appropriate biotinylated secondary antibodies (Vectastain Elite Kit, Vector Laboratories, Burlingame, CA, USA) as described previously. ${ }^{13}$ Peroxidase reaction product was visualised using 3,3'-diaminobenzidine (Sigma, Deisenhofen, Germany) as chromogene and $\mathrm{H}_{2} \mathrm{O}_{2}$ as cosubstrate. All immunohistochemical studies were performed on paraffin-embedded specimen sections. The respective primary antibodies are listed in table 1.

Breast carcinoma and tonsillar tissue served as positive controls. For quantitative analysis, 10 high-power fields (HPFs) of cross sections of each sural nerve were evaluated. Inflammatory infiltrates were depicted by leucocyte common antigen (LCA) staining. Epineurial, perivascular and intramural LCA-positive, small, mature lymphocytes were counted. In immunohistochemically stained sections for the antigens listed in table 1, epineurial, perivascular and intramural immunoreactive cells were counted. Their numbers were correlated with epineurial, perivascular and intramural small, mature lymphocytes depicted by hemalum counterstaining, which were not reactive in the respective immunostainings. Mean $\pm S D$ of the total number of immunoreactive cells of the respective antigens as well as the percentage of the sum of immunoreactive and non-immunoreactive epineurial, perivascular and intramural small, mature lymphocytes of the respective antigens were evaluated for each antigen on the respective slide in $10 \mathrm{HPF}$.

\section{Statistical analysis}

Comparisons between clinical and electrophysiological tests and frequency of inflammatory infiltrates were performed using the Mann-Whitney U test; $p$ values of $<0.05$ were considered statistically significant.

\section{RESULTS}

\section{Patients and clinical studies}

Mean age of the patients and controls at the time of biopsy was 67 and 61 years, respectively. The time interval between onset of clinical symptoms and definite diagnosis varied from 1 to 20 years (mean: 4.85 years). $90 \%$ of the patients presented with sensory and motor deficits; the remaining patients showed sensory symptoms only. Electrophysiological studies, comprising distal motor latencies, proximal and distal compound muscle action potential amplitudes, and motor nerve conduction velocity of the right tibial or peroneal nerve as well as sensory nerve action potential amplitudes, and sensory nerve conduction velocity of the right sural nerve, revealed severe axonal damage of sensory and motor nerve fibres in all patients (data not shown). Patients with a long history of symptoms showed no distinct clinical, electrophysiological or neuropathological changes compared with patients with short duration of symptoms. Therefore, duration of disease influenced neither disease severity nor the extent of inflammatory infiltrates.

\section{Immunohistochemical profiling}

NSVN sural nerves of all patients (20/20, 100\%) harboured epineurial, perivascular or intramural LCA + inflammatory infiltrates (mean \pm SD/10 HPF: 225.8 \pm 66.24 LCA+ cells; table 2).

Vascular wall damage had led to haemorrhage with hemosiderin deposits in 60\% (12/20) of patients. Asymmetric loss of nerve fibres was present in $80 \%$ of patients $(16 / 20$, figure 1$)$. $\mathrm{LCA}+$ cells were confined to the epineurial tissue of $3 / 5$ control patients (mean $\pm \mathrm{SD}: 1.6 \pm 1.52 \mathrm{LCA}+$ cells). Epineurial infiltrates consisted predominantly of $\mathrm{CD} 8+$ and some CD4+, $\mathrm{CD} 3+\mathrm{T}$ cells as well as some CD68 + macrophages. Endoneurial leucocytes corresponded to $\mathrm{CD} 68+$ macrophages. In controls, LCA+ cells were absent from vessels.

The sum of immunoreactive and non-immunoreactive lymphocytes in 10 HPFs of the cross-orientated sural nerve specimens for each antigen strongly correlated with the number 
Neuromuscular

Table 2 Total number of epineurial, perivascular or intramural small, mature lymphocytes in 10 HPF in NSVN sural nerve biopsies

\begin{tabular}{lll}
\hline & Mean & SD \\
\hline LCA+ & 225.75 & 66.24 \\
h+CD52+ and h+CD52- & 222.3 & 65.93 \\
h+CD25+ and h+CD25- & 224.75 & 65.48 \\
h+BAFF+ and h+BAFF- & 217.75 & 59.34 \\
$h+C D 20+$ and h+CD20- & 223.3 & 69.99 \\
$h+C D 49 d+$ and h+CD49d- & 221.95 & 70.05 \\
$h+C D 126+, h+C D 126-$ & 222.9 & 69.92 \\
\hline
\end{tabular}

$\mathrm{h}+$, hemalum positive; $\mathrm{HPF}$, high power field; LCA, leucocyte common antigen; NSVN, non-systemic vasculitic neuropathy.

of epineurial, perivascular or intramural LCA-positive, small, mature lymphocytes (table 2). CD52 was the most abundantly expressed antigen on epineurial lymphocytes in NSVN biopsies and was present in all specimens (20/20, figure 2, table 3$)$. There was no difference in the CD52 expression profile depending on localisation of lymphocytes in the epineurial tissue, that is, epineurial perivascular or intramural.

Sural nerve biopsies of patients with AASV (2/2) harboured large infiltrates of LCA+ vessel-associated lymphocytes (mean \pm SD/10 HPF: $445.5 \pm 86.76$ ) predominantly consisting of $\mathrm{CD} 3+\mathrm{T}$ cells. Vessel-associated infiltrates comprised numerous CD52+ lymphocytes (mean \pm SD/10 HPF: $224.5 \pm 34.48$; corresponding to $50.39 \%$ of all lymphocytes). The CD52 epitope was only weakly expressed on single intramural lymphocytes in the biopsies of two patients with temporal arteritis and completely absent from nerve biopsies of two patients with CIDP (data not shown). Sural nerves of all patients with NSVN (20/20) harboured $\mathrm{BAFF}+$ vessel-associated lymphocytes (table 3). Although $85 \%(17 / 20)$ and 50\% (10/20) of the patients with NSVN showed an expression of the CD25 and CD20 antigen, respectively, these antigens were confined to a few lymphocytes only (table 3). CD126 was expressed on single perivascular lymphocytes in $15 \%(3 / 20)$ of the patients with NSVN only (table 3). The expression profile of these antigens did not show any correlation neither with clinical nor with electrophysiological parameters.

\section{DISCUSSION}

Our data demonstrate that in NSVN, lymphocytes express various surface molecules and cytokines for which therapeutic monoclonal antibodies are available. As such, to the best of our knowledge, this is the first study that provides evidence for the expression of therapeutically targetable surface molecules in NSVN.

At the time point of biopsy, patients exhibited neurological symptoms requiring treatment. These clinical signs of disease activity were associated with the presence of lymphocytic infiltration in the epineurial tissue. Since the inflammatory infiltrates in NSVN are by definition restricted to the peripheral nervous system, our approach of immunophenotyping nerve biopsy specimens is the only one that allows identification of treatment targets. The lack of a correlation between clinical data and antigen expression profiles in the target organ, that is, the peripheral nerve, further emphasises the requirement of nerve biopsy analysis to guide novel concepts for treating NSVN.

Interestingly, the CD52 antigen, which is expressed by a variety of immune cells including $\mathrm{T}$ and $\mathrm{B}$ cells as well as macrophages, was expressed in all NSVN biopsies. Moreover, CD52
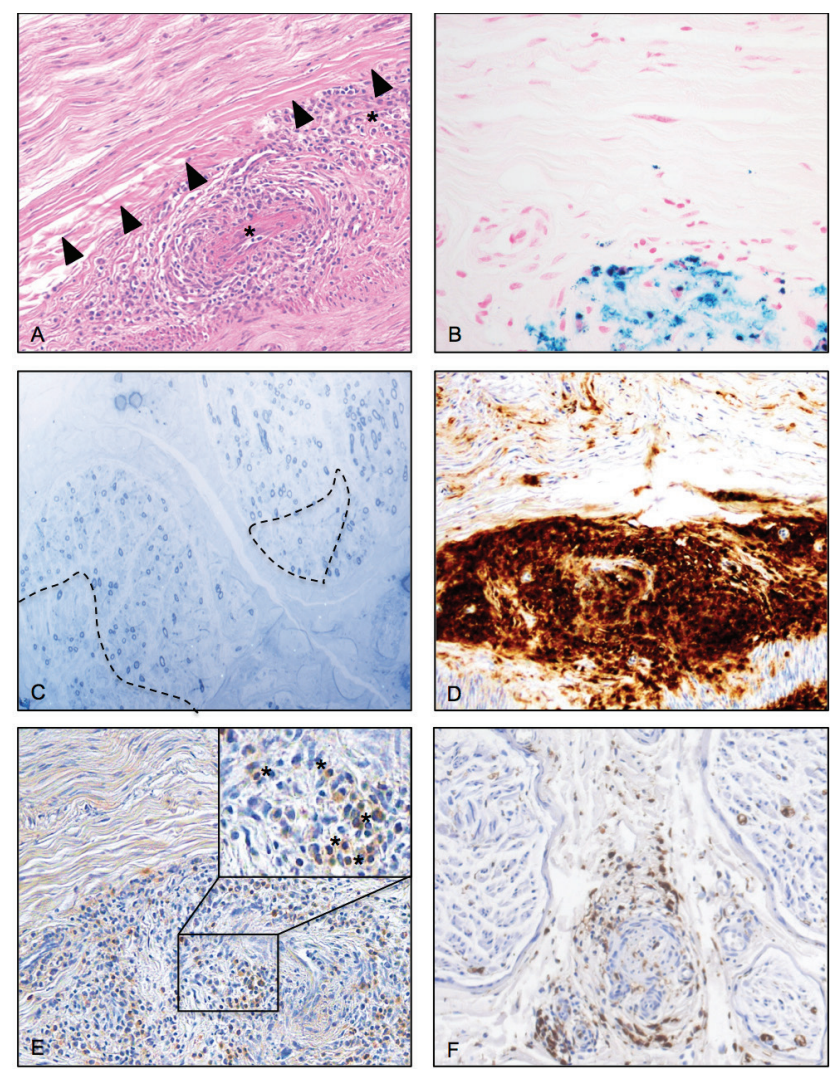

Figure 1 Histochemical and immunohistochemical characterisation of sural nerve vasculitis. (A) The vessel wall and the perivascular area of two epineurial blood vessels (asterisks) adjacent to nerve fascicles (arrowheads) in the sural nerve harbour numerous inflammatory infiltrates, which predominantly consist of small, mature lymphocytes. (B) The vessel wall infiltrated by leucocytes as well as the adjacent epineurial tissue in the sural nerve harbours hemosiderin deposits indicative of previous haemorrhage subsequent to inflammation. (C) Sector-like loss (dotted lines) of nerve fibres in sural nerve fascicles. The two fascicles differ in severity of nerve fibre loss with better preservation of nerve fibres in the upper right fascicle. (D) Many leucocyte common antigen (LCA)-positive leucocytes have infiltrated the wall of a medium-sized vessel and the adjacent epineurium. In contrast, only few LCA-positive leucocytes with elongated cytoplasm and small processes, thus, corresponding to macrophages, have infiltrated the endoneurium. (E) The vasculitic infiltrate predominantly consists of small CD3-positive T cells (asterisks insert E). (F) Only a few CD68+ macrophages contribute to epineurial vessel-associated infiltrates, whereas the majority of endoneurial leucocytes correspond to foamy macrophages indicating progress of neuropathy. (A) H\&E staining; original magnification x200; (B) Prussian blue staining; original magnification $x 400 ;(C)$ resin-embedded semithin sections; toluidine blue; original magnification $\mathrm{x} 200$; (D) immunohistochemistry with mouse antihuman LCA and slight counterstain with hemalum; original magnification x200; (E) immunohistochemistry with mouse anti-human CD3 and slight counterstain with hemalum; original magnification $\times 200$; insert $\times 400$; (F) immunohistochemistry with mouse anti-human CD68 and slight counterstain with hemalum; original magnification x200.

was the most frequently detected antigen (21.49\%) in inflammatory infiltrates.

CD52 can be targeted by the humanised monoclonal antibody alemtuzumab, which has been approved for the treatment of patients with multiple sclerosis. ${ }^{9}$ Our results suggest that CD52 may be a suitable target also in NSVN. Expression of the CD52 antigen was not confined to NSVN but was also detected in 

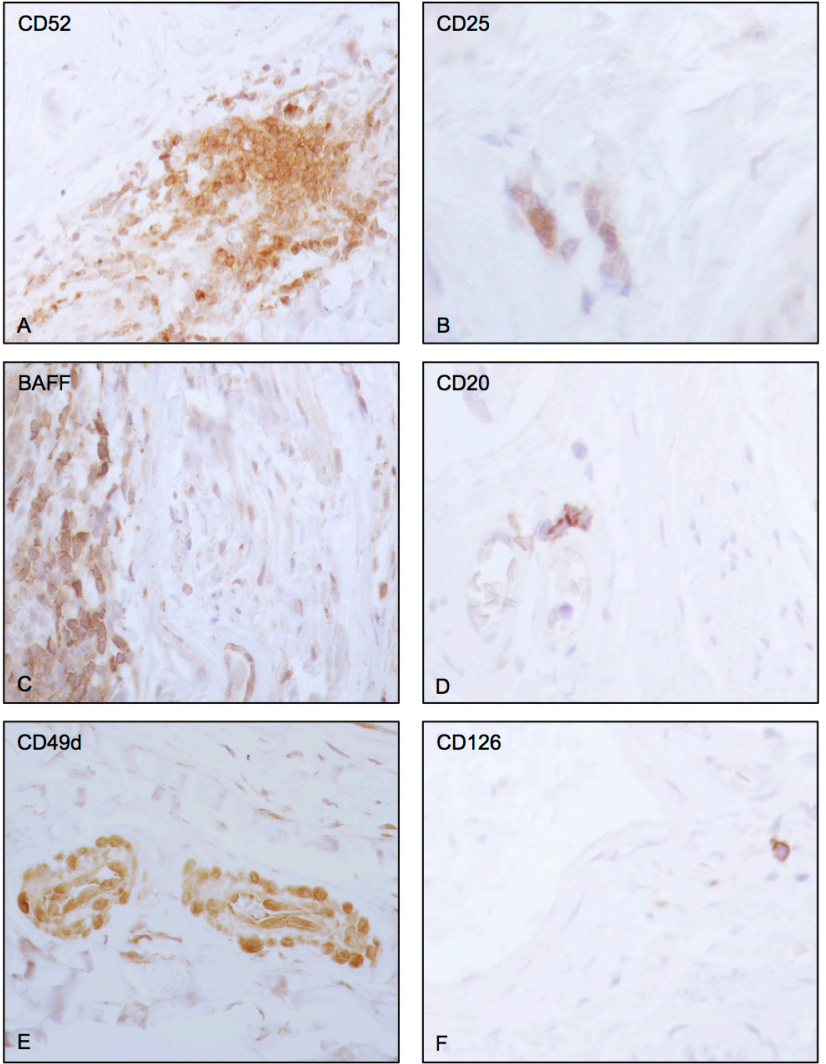

Figure 2 Expression of the CD52, CD25, BAFF, CD20, CD49d and CD126 antigens in vasculitic infiltrates of the sural nerve. (A) The sural nerve harbours numerous CD52-positive leucocytes, which have infiltrated the vessel wall and the adjacent epineurium. (B) Vessel-associated CD25positive leucocytes are present. (C) Numerous BAFF-positive leucocytes have infiltrated the walls of epineurial blood vessels and the epineurium. (D) Only single CD20-positive B cells are present. (E) Some medium-sized vessel walls in the epineurium have been invaded by CD49d-positive cells. (F) Only single CD126-positive leucocytes are present. (A-F) Immunohistochemistry with rat anti-human CD52 (A), mouse anti-human CD25 (B), rat anti-human BAFF (C), mouse anti-human CD20 (D), rabbit anti-human CD49d (E) and mouse anti-human CD126 (F) and slight counterstain with hemalum; original magnification $\times 400$.

patients with AASV (this study). In the study by Walsh et al ${ }^{14}$ and in a study with Behcet's disease ${ }^{15}$ patients were responsive to anti-CD52 treatment. Therefore, such an approach may extend the use of alemtuzumab in patients with AASV, in whom beneficial effects on the overall outcome were suggested. ${ }^{14}$ However, in the latter study, neuropathic symptoms were not specifically assessed. In other inflammatory conditions, for example, in

Table 3 Numbers of antigen-expressing perivascular or intramural, epineurial lymphocytes in $10 \mathrm{HPF}$ in NSVN sural nerve biopsies.

\begin{tabular}{lllc}
\hline & Mean & SD & $\begin{array}{l}\text { Percent of all } \\
\text { lymphocytes }\end{array}$ \\
\hline CD52 & 51.6 & 38.97 & 21.49 \\
CD25 & 13.1 & 11.96 & 5.22 \\
BAFF & 25.85 & 21.12 & 11.25 \\
CD20 & 12.75 & 35.13 & 5.84 \\
CD49d & 26.9 & 22.89 & 10.99 \\
CD126 & 0.55 & 1.5 & 0.15 \\
\hline
\end{tabular}

HPF, high power field; NSVN, non-systemic vasculitic neuropathy.
CIDP (this study and the study by Marsh et $a l^{16}$ ), temporal arteritis (this study) and inflammatory orbital lesions, ${ }^{17}$ CD52-expressing lymphocytes or response to anti-CD52 treatment were either absent or rare, thus, not supporting anti-CD52 target treatment in these disorders.

BAFF (11.25\%) and CD49d (10.99\%) were expressed by significant populations of leucocytes. These observations may warrant the application of belimumab, a monoclonal antibody targeting BAFF, which has been used in the therapy of systemic vasculitis. In systemic lupus erythematodes, two phase III studies demonstrated efficacy in reducing overall disease activity; however, frequency of vasculitic neuropathy at baseline and outcome on neuropathic symptoms were not reported. ${ }^{18-20}$

Furthermore, our data support the idea to apply natalizumab in NSVN, a monoclonal antibody targeting CD49d. Natalizumab is currently used in highly active multiple sclerosis, in which it has been shown to reduce the risk of disease progression and of clinical relapses. In individual patients with CIDP, natalizumab treatment led to an improvement and stabilisation in two and one patient(s), respectively, while disease progressed in one patient. $^{2122}$

The sural nerve of patients suffering from NSVN harboured only few B cells, which is in accordance with a previous study. ${ }^{23}$ In summary, we have, for the first time, identified immune molecules in the sural nerve of patients with NSVN which are potentially targetable. Molecular pathogenesis and the precise contribution of these molecules to NSVN remain to be elucidated. Data suggest that targeting the CD52 antigen, for example, by alemtuzumab, may constitute a promising novel treatment approach in NSVN. Results warrant future controlled trials in NSVN with monoclonal antibodies.

Correction notice This paper has been amended since it was published Online First. Owing to a scripting error, some of the publisher names in the references were replaced with 'BMJ Publishing Group'. This only affected the full text version, not the PDF. We have since corrected these errors and the correct publishers have been inserted into the references.

Acknowledgements The expert technical assistance of Mariana Carstov is gratefully acknowledged.

Contributors CS: Acquisition and analysis of data, drafting the manuscript for content. GW: Study concept, drafting the manuscript for content. JB: drafting the manuscript for content, data analysis. GRF: Study concept, drafting the manuscript for content. MD: Study concept, drafting the manuscript for content. AB: Acquisition and analysis of data, study concept, drafting the manuscript for content. HCL: study design and concept, drafting the manuscript for content.

Competing interests $\mathrm{HCL}$ has received personal compensations and/or grant support from Baxter, Celgene, CSL Behring, Fresenius, Genzyme, Grifols and Novartis.

Provenance and peer review Not commissioned; externally peer reviewed.

(C) Article author(s) (or their employer(s) unless otherwise stated in the text of the article) 2017. All rights reserved. No commercial use is permitted unless otherwise expressly granted.

\section{REFERENCES}

1 Collins MP, Dyck PJ, Gronseth GS, et al. Peripheral Nerve Society. Peripheral Nerve Society Guideline on the classification, diagnosis, investigation, and immunosuppressive therapy of non-systemic vasculitic neuropathy: executive summary. J Peripher Nerv Syst 2010;15:176-84.

2 Jayne D, Rasmussen N, Andrassy K, et al. European Vasculitis Study Group. A randomized trial of maintenance therapy for vasculitis associated with antineutrophil cytoplasmic autoantibodies. N Eng/ J Med 2003;349:36-44.

3 Pagnoux C, Mahr A, Hamidou MA, et al. French Vasculitis Study Group. Azathioprine or methotrexate maintenance for ANCA-associated vasculitis. N Engl J Med 2008;359:2790-803.

4 Sugiura M, Koike $H$, lijima M, et al. Clinicopathologic features of nonsystemic vasculitic neuropathy and microscopic polyangiitis-associated neuropathy: a comparative study. J Neurol Sci 2006;241:31-7.

5 Üçeyler N, Geng A, Reiners K, et al. Non-systemic vasculitic neuropathy: single-center follow-up of 60 patients. J Neurol 2015;262:2092-100. 
6 Collins MP, Periquet MI, Mendell JR, et al. Nonsystemic vasculitic neuropathy: insights from a clinical cohort. Neurology 2003;61:623-30.

7 Hauser SL, Waubant E, Arnold DL, et al. B-Cell depletion with rituximab in relapsingremitting multiple sclerosis. N Engl J Med 2008;358:676-88.

8 Cohen JA, Coles AJ, Arnold DL, et al. Alemtuzumab versus interferon beta 1a as firstline treatment for patients with relapsing-remitting multiple sclerosis: a randomised controlled phase 3 trial. Lancet 2012;380:1819-28.

9 Coles AJ, Twyman CL, Arnold DL, et al. Alemtuzumab for patients with relapsing multiple sclerosis after disease-modifying therapy: a randomised controlled phase 3 trial. Lancet 2012;380:1829-39.

10 Kappos L, Wiendl H, Selmaj K, et al. Daclizumab HYP versus interferon Beta-1a in relapsing multiple sclerosis. N Engl J Med 2015;373:1418-28.

11 Araki M, Matsuoka T, Miyamoto K, et al. Efficacy of the anti-IL-6 receptor antibody tocilizumab in neuromyelitis optica: a pilot study. Neurology 2014;82:1302-6.

12 Kimura J. Electrodiagnosis in diseases of nerve and muscle: principles and practice. Oxford University Press, 2013.

13 Brunn A, Hans VJ, Vogelgesang S, et al. Inflammatory myopathy with abundant macrophages and dermatomyositis: two stages of one disorder or two distinct entities? Acta Neuropathol 2009;118:793-801.

14 Walsh M, Chaudhry A, Jayne D. Long-term follow-up of relapsing/refractory antineutrophil cytoplasm antibody associated vasculitis treated with the lymphocyte depleting antibody alemtuzumab (CAMPATH-1H). Ann Rheum Dis 2008;67:1322-7.

15 Lockwood CM, Hale G, Waldman H, et al. Remission induction in Behçet's disease following lymphocyte depletion by the anti-CD52 antibody CAMPATH 1-H.

Rheumatology 2003;42:1539-44.
16 Marsh EA, Hirst CL, Llewelyn JG, et al. Alemtuzumab in the treatment of IVIGdependent chronic inflammatory demyelinating polyneuropathy. J Neurol 2010;257:913-9.

17 Ho VH, Chevez-Barrios P, Jorgensen JL, et al. Receptor expression in orbital inflammatory syndromes and implications for targeted therapy. Tissue Antigens 2007;70:105-9.

18 Manzi S, Sánchez-Guerrero J, Merrill JT, et al. Effects of belimumab, a B lymphocyte stimulator-specific inhibitor, on disease activity across multiple organ domains in patients with systemic lupus erythematosus: combined results from two phase III trials. Ann Rheum Dis 2012;71:1833-8.

19 Furie R, Petri M, Zamani O, et al. A phase III, randomized, placebo-controlled study of Belimumab, a monoclonal antibody that inhibits B lymphocyte stimulator, in patients with systemic lupus erythematosus. Arthritis Rheum 2011;63:3918-30.

20 Navarra SV, Guzmán RM, Gallacher AE, et al. Efficacy and safety of belimumab in patients with active systemic lupus erythematosus: a randomised, placebo-controlled, phase 3 trial. Lancet 2011;377:721-31.

21 Vallat JM, Mathis S, Ghorab K, et al. Natalizumab as a disease-modifying therapy in chronic inflammatory demyelinating polyneuropathy - a report of three cases. Eur Neurol 2015;73:294-302.

22 Wolf $\mathrm{C}$, Menge T, Stenner MP, et al. Natalizumab treatment in a patient with chronic inflammatory demyelinating polyneuropathy. Arch Neurol 2010;67:881-3.

23 Vrancken AF, Notermans NC, Jansen GH, et al. Progressive idiopathic axonal neuropathy - a comparative clinical and histopathological study with vasculitic neuropathy. J Neurol 2004;251:269-78. 

monoclonal antibodies in non-systemic vasculitic neuropathy

Christian Schneider, Gilbert Wunderlich, Johannes Bleistein, Gereon R

Fink, Martina Deckert, Anna Brunn and Helmar Christoph Lehmann

J Neurol Neurosurg Psychiatry 2017 88: 756-760 originally published online May 26, 2017

doi: 10.1136/jnnp-2017-315878

Updated information and services can be found at:

http://jnnp.bmj.com/content/88/9/756

References

Email alerting service
These include:

This article cites 22 articles, 2 of which you can access for free at: http://jnnp.bmj.com/content/88/9/756\#BIBL

Receive free email alerts when new articles cite this article. Sign up in the box at the top right corner of the online article.

\section{Notes}

To request permissions go to:

http://group.bmj.com/group/rights-licensing/permissions

To order reprints go to:

http://journals.bmj.com/cgi/reprintform

To subscribe to BMJ go to:

http://group.bmj.com/subscribe/ 